International Journal of Pure and Applied Mathematics

Volume 94 No. $1 \quad 2014,89-99$

ISSN: 1311-8080 (printed version); ISSN: 1314-3395 (on-line version)

url: http://www.ijpam.eu

doi: http://dx.doi.org/10.12732/ijpam.v94i1.10

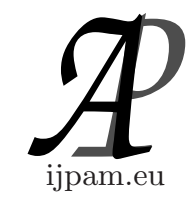

\title{
THE FUNCTORIAL RELATIONS BETWEEN \\ ALEXANDROV FUZZY TOPOLOGIES AND UPPER APPROXIMATION OPERATORS
}

\author{
Yong Chan Kim \\ Department of Mathematics \\ Gangneung-Wonju University \\ Gangneung, Gangwondo, 210-702, KOREA
}

\begin{abstract}
In this paper, we investigate functorial relations between Alexandrov fuzzy topologies and upper approximation operators in complete residuated lattices. We present some examples.
\end{abstract}

AMS Subject Classification: 03E72, 03G10, 06A15, 06F07, 54A40

Key Words: complete residuated lattices, fuzzy preorders, upper approximation operators, Alexandrov fuzzy topologies

\section{Introduction}

The relationship between rough set theory and topological spaces was investigated in sets [8]. Hájek [2] introduced a complete residuated lattice which is an algebraic structure for many valued logic. It is an important mathematical tool for algebraic structure of fuzzy contexts [1-7, 9,10, 13-16]. Höhle [3] introduced $L$-fuzzy topologies and $L$-fuzzy interior operators in complete residuated lattices. Kim [5-7] investigated the properties of upper approximation operators induced by Alexandrov fuzzy topologies in complete residuated lattices in a sense as Höhle's $L$-fuzzy topologies and $L$-fuzzy interior operators.

Received: May 3, 2014

(c) 2014 Academic Publications, Ltd. url: www.acadpubl.eu 
In this paper, we investigate functorial relations between Alexandrov fuzzy topologies and upper approximation operators in complete residuated lattices. We give their examples.

\section{Preliminaries}

Definition 2.1. [1-3] A structure $(L, \vee, \wedge, \odot, \rightarrow, \perp, \top)$ is called a complete residuated lattice iff it satisfies the following properties:

(L1) $(L, \vee, \wedge, \perp, \top)$ is a complete lattice where $\perp$ is the bottom element and $\top$ is the top element;

(L2) $(L, \odot, \top)$ is a commutative monoid;

(L3) an adjointness property holds,i.e., for all $x, y, z \in X$,

$$
x \leq y \rightarrow z \text { iff } x \odot y \leq z .
$$

A operator ${ }^{*}: L \rightarrow L$ defined by $a^{*}=a \rightarrow \perp$ is called strong negations if $a^{* *}=a$. For $\alpha \in L, A \in L^{X}$, we denote $(\alpha \rightarrow A),(\alpha \odot A), \bar{\alpha}, \top_{x}, \top_{x}^{*} \in L^{X}$ as

$$
\begin{gathered}
(\alpha \rightarrow A)(x)=\alpha \rightarrow A(x),(\alpha \odot A)(x)=\alpha \odot A(x), \bar{\alpha}(x)=\alpha, \\
\top_{x}(y)=\left\{\begin{array}{ll}
\top, & \text { if } y=x, \\
\perp, & \text { otherwise, }
\end{array} \top_{x}^{*}(y)= \begin{cases}\perp, & \text { if } y=x, \\
\top, & \text { otherwise } .\end{cases} \right.
\end{gathered}
$$

In this paper, we assume that $\left(L, \vee, \wedge, \odot, \rightarrow,^{*}, \perp, \top\right)$ be a complete residuated lattice with a strong negation *.

Definition 2.2. $[9,10]$ Let $X$ be a set. A function $R_{X}: X \times X \rightarrow L$ is called a fuzzy preorder if it satisfies the following conditions:

(E1) $R_{X}(x, x)=\top$ for all $x \in X$,

(E2) $R_{X}(x, y) \odot R_{X}(y, z) \leq R_{X}(x, z)$, for all $x, y, z \in X$.

Example 2.3. (1) We define a function $R_{L}: L \times L \rightarrow L$ as $R_{L}(x, y)=$ $x \rightarrow y$. Then $\left(L, R_{L}\right)$ is a fuzzy preordered set.

(2) We define a function $e_{L^{X}}: L^{X} \times L^{X} \rightarrow L$ as $e_{L^{X}}(A, B)=\bigwedge_{x \in X}(A(x) \rightarrow$ $B(x))$. Then $\left(L^{X}, e_{L^{X}}\right)$ is a fuzzy preordered set.

Definition 2.4. [4-7,9] An operator $\mathbf{T}: L^{X} \rightarrow L$ is called an Alexander fuzzy topology on $X$ iff it satisfies the following conditions: for all $\alpha \in L, A, A_{i} \in$ $L^{X}$ 
(T1) $\mathbf{T}(\bar{\alpha})=\top$,

(T2) $\mathbf{T}\left(\bigwedge_{i \in \Gamma} A_{i}\right) \geq \bigwedge_{i \in \Gamma} \mathbf{T}\left(A_{i}\right)$ and $\mathbf{T}\left(\bigvee_{i \in \Gamma} A_{i}\right) \geq \bigwedge_{i \in \Gamma} \mathbf{T}\left(A_{i}\right)$,

(T3) $\mathbf{T}(\alpha \odot A) \geq \mathbf{T}(A)$,

(T4) $\mathbf{T}(\alpha \rightarrow A) \geq \mathbf{T}(A)$.

A map $f:\left(X, \mathbf{T}_{X}\right) \rightarrow\left(Y, \mathbf{T}_{Y}\right)$ is fuzzy continuous if $\mathbf{T}_{X}\left(f^{-1}(B)\right) \geq \mathbf{T}_{Y}(B)$ for all $B \in L^{Y}$.

Definition 2.5. [5-7] A map $\mathcal{H}: L^{X} \rightarrow L^{X}$ is called an upper approximation operator iff it satisfies the following conditions

(H1) $\mathcal{H}(\alpha \odot A)=\alpha \odot \mathcal{H}(A)$ for all $A \in L^{X}$ and $\alpha \in L$.

(H2) $\mathcal{H}\left(\bigvee_{i \in I} A_{i}\right)=\bigvee_{i \in I} \mathcal{H}\left(A_{i}\right)$ for all $A_{i} \in L^{X}$.

(H3) $A \leq \mathcal{H}(A)$,

(H4) $\mathcal{H}(\mathcal{H}(A)) \leq \mathcal{H}(A)$, for all $A \in L^{X}$.

Lemma 2.6. [1-3] Let $\left(L, \vee, \wedge, \odot, \rightarrow,^{*}, \perp, \top\right)$ be a complete residuated lattice with a strong negation ${ }^{*}$. For each $x, y, z, x_{i}, y_{i} \in L$, the following properties hold.

(1) If $y \leq z$, then $x \odot y \leq x \odot z$.

(2) If $y \leq z$, then $x \rightarrow y \leq x \rightarrow z$ and $z \rightarrow x \leq y \rightarrow x$.

(3) $x \rightarrow y=\top$ iff $x \leq y$.

(4) $x \rightarrow \top=\top$ and $\top \rightarrow x=x$.

(5) $x \odot y \leq x \wedge y$.

(6) $x \odot\left(\bigvee_{i \in \Gamma} y_{i}\right)=\bigvee_{i \in \Gamma}\left(x \odot y_{i}\right)$ and $\left(\bigvee_{i \in \Gamma} x_{i}\right) \odot y=\bigvee_{i \in \Gamma}\left(x_{i} \odot y\right)$.

(7) $x \rightarrow\left(\bigwedge_{i \in \Gamma} y_{i}\right)=\bigwedge_{i \in \Gamma}\left(x \rightarrow y_{i}\right)$ and $\left(\bigvee_{i \in \Gamma} x_{i}\right) \rightarrow y=\bigwedge_{i \in \Gamma}\left(x_{i} \rightarrow y\right)$. $\left.y_{i}\right)$.

(8) $\bigvee_{i \in \Gamma} x_{i} \rightarrow \bigvee_{i \in \Gamma} y_{i} \geq \bigwedge_{i \in \Gamma}\left(x_{i} \rightarrow y_{i}\right)$ and $\bigwedge_{i \in \Gamma} x_{i} \rightarrow \bigwedge_{i \in \Gamma} y_{i} \geq \bigwedge_{i \in \Gamma}\left(x_{i} \rightarrow\right.$

(9) $(x \rightarrow y) \odot x \leq y$ and $(y \rightarrow z) \odot(x \rightarrow y) \leq(x \rightarrow z)$.

(10) $x \rightarrow y \leq(y \rightarrow z) \rightarrow(x \rightarrow z)$ and $x \rightarrow y \leq(z \rightarrow x) \rightarrow(z \rightarrow y)$.

(11) $\bigwedge_{i \in \Gamma} x_{i}^{*}=\left(\bigvee_{i \in \Gamma} x_{i}\right)^{*}$ and $\bigvee_{i \in \Gamma} x_{i}^{*}=\left(\bigwedge_{i \in \Gamma} x_{i}\right)^{*}$

(12) $(x \odot y) \rightarrow z=x \rightarrow(y \rightarrow z)=y \rightarrow(x \rightarrow z)$ and $(x \odot y)^{*}=x \rightarrow y^{*}$.

(13) $x^{*} \rightarrow y^{*}=y \rightarrow x$ and $(x \rightarrow y)^{*}=x \odot y^{*}$.

(14) $y \rightarrow z \leq x \odot y \rightarrow x \odot z$.

(15) $x \rightarrow y \odot z \geq(x \rightarrow y) \odot z$.

Theorem 2.7. [5-7] Let $\mathbf{T}: L^{X} \rightarrow L$ be an Alexander fuzzy topology. Define $\mathbf{T}^{*}(A)=\mathbf{T}\left(A^{*}\right)$. Then $\mathbf{T}^{*}$ is an Alexander fuzzy topology.

Theorem 2.8. [6] Let $\mathbf{T}_{X}$ be an Alexandrov fuzzy topology on $X$. Define $\mathcal{H}_{T_{X}}: L^{X} \times L \rightarrow L^{X}$ as follows

$$
\mathcal{H}_{T_{X}}(A, r)=\bigwedge\left\{B \in L^{X} \mid A \leq B, \mathbf{T}_{X}(B) \geq r^{*}\right\}
$$


Then we have the following properties.

(1) $\mathcal{H}_{T_{X}}(-, r): L^{X} \rightarrow L^{X}$ is an upper approximation operator.

(2) If $r \leq s$, then $\mathcal{H}_{T_{X}}(A, s) \leq \mathcal{H}_{T_{X}}(A, r)$ for all $A \in L^{X}$.

(3) There exists a fuzzy preorder $R_{T_{X}}^{r} \in L^{X \times X}$ such that

$$
\mathcal{H}_{T_{X}}(A, r)=\bigvee_{x \in X}\left(A(x) \odot R_{T_{X}}^{r}(x, y)\right) \text {. }
$$

(4) If $r \leq s$, then $R_{T_{X}}^{r} \geq R_{T_{X}}^{s}$ for all $A \in L^{X}$.

(5) If $\mathcal{H}_{T_{X}}\left(A, r_{i}\right)=B$ for all $i \in \Gamma \neq \emptyset$, then $\mathcal{H}_{T_{X}}\left(A, \bigwedge_{i \in \Gamma} r_{i}\right)=B$.

(6) Define $\mathbf{T}_{H_{T_{X}}}: L^{X} \rightarrow L$ as

$$
\mathbf{T}_{H_{T_{X}}}(A)=\bigvee\left\{r^{*} \in L \mid \mathcal{H}_{T_{X}}(A, r)=A\right\}
$$

Then $\mathbf{T}_{H_{T_{X}}}=\mathbf{T}_{X}$ is an Alexandrov fuzzy topology on $X$.

(7) There exists an Alexandrov fuzzy topology $\mathbf{T}_{T_{X}}^{r}$ such that

$$
\mathbf{T}_{T_{X}}^{r}(A)=e_{L^{X}}\left(\mathcal{H}_{T_{X}}(A, r), A\right) .
$$

(8) If $r \leq s$, then $\mathbf{T}_{T_{X}}^{r} \leq \mathbf{T}_{T_{X}}^{s}$ for all $A \in L^{X}$.

(9) Define $\mathbf{T}_{T_{X}}: L^{X} \rightarrow L$ as

$$
\mathbf{T}_{T_{X}}(A)=\bigvee\left\{r^{*} \in L \mid \mathbf{T}_{T_{X}}^{r}(A)=\top\right\} .
$$

Then $\mathbf{T}_{T_{X}}=\mathbf{T}_{X}=\mathbf{T}_{H_{T_{X}}}$ is an Alexandrov fuzzy topology on $X$.

\section{The functorial relations between Alexandrov fuzzy topologies and upper approximation operators}

Theorem 3.1 Let $\mathbf{T}_{X}$ and $\mathbf{T}_{Y}$ be an Alexandrov fuzzy topologies on $X$ and $Y$, respectively. Let $f: X \rightarrow Y$ be a map. Then the following statements are equivalent.

(1) $f:\left(X, \mathbf{T}_{X}\right) \rightarrow\left(Y, \mathbf{T}_{Y}\right)$ is fuzzy continuous.

(2) $f:\left(X, \mathbf{T}_{X}^{*}\right) \rightarrow\left(Y, \mathbf{T}_{Y}^{*}\right)$ is fuzzy continuous.

(3) $f^{-1}\left(\mathcal{H}_{T_{Y}}\left(\top_{f(x)}, r\right)\right) \geq \mathcal{H}_{T_{X}}\left(\top_{x}, r\right)$ for all $x \in X$ and $r \in L$.

(4) $f^{-1}\left(\mathcal{H}_{T_{Y}^{*}}\left(\top_{f(x)}, r\right)\right) \geq \mathcal{H}_{T_{X}^{*}}\left(\top_{x}, r\right)$ for all $x \in X$ and $r \in L$.

(5) There exists fuzzy preorders $R_{T_{X}}^{r} \in L^{X \times X}$ and $R_{T_{Y}}^{r} \in L^{Y \times Y}$ such that, for all $x, y \in X$,

$$
R_{T_{X}}^{r}(x, y) \leq R_{T_{Y}}^{r}(f(x), f(y))
$$


(6) There exists fuzzy preorders $R_{T_{X}^{*}}^{r} \in L^{X \times X}$ and $R_{T_{Y}^{*}}^{r} \in L^{Y \times Y}$ such that, for all $x, y \in X$,

$$
R_{T_{X}^{*}}^{r}(x, y) \leq R_{T_{Y}^{*}}^{r}(f(x), f(y)) .
$$

(7) $f^{-1}\left(\mathcal{H}_{T_{Y}}(B, r)\right) \geq \mathcal{H}_{T_{X}}\left(f^{-1}(B), r\right)$ for all $B \in L^{Y}$ and $r \in L$.

(8) $f^{-1}\left(\mathcal{H}_{T_{Y}^{*}}(B, r)\right) \geq \mathcal{H}_{T_{X}^{*}}\left(f^{-1}(B), r\right)$ for all $B \in L^{Y}$ and $r \in L$.

(9) $f\left(\mathcal{H}_{T_{X}}(A, r)\right) \leq \mathcal{H}_{T_{Y}}(f(A), r)$ for all $A \in L^{X}$ and $r \in L$.

(10) $f\left(\mathcal{H}_{T_{X}^{*}}(A, r)\right) \leq \mathcal{H}_{T_{Y}^{*}}(f(A), r)$ for all $A \in L^{X}$ and $r \in L$.

(11) $\mathbf{T}_{T_{X}}^{r}\left(f^{-1}(B)\right) \geq \mathbf{T}_{T_{Y}}^{r}(B)$ for all $B \in L^{Y}$ and $r \in L$.

(12) $\mathbf{T}_{T_{X}^{*}}^{r}\left(f^{-1}(B)\right) \geq \mathbf{T}_{T_{Y}^{*}}^{r}(B)$ for all $B \in L^{Y}$ and $r \in L$.

(13) $\mathbf{T}_{\mathcal{H}_{T_{X}}}\left(f^{-1}(B)\right) \geq \mathbf{T}_{\mathcal{H}_{T_{Y}}}(B)$ for all $B \in L^{Y}$.

(14) $\mathbf{T}_{\mathcal{H}_{T_{X}^{*}}}\left(f^{-1}(B)\right) \geq \mathbf{T}_{\mathcal{H}_{T_{Y}^{*}}}(B)$ for all $B \in L^{Y}$.

(15) $\mathbf{T}_{T_{X}}\left(f^{-1}(B)\right) \geq \mathbf{T}_{T_{Y}}(B)$ for all $B \in L^{Y}$.

(16) $\mathbf{T}_{T_{X}^{*}}\left(f^{-1}(B)\right) \geq \mathbf{T}_{T_{Y}^{*}}(B)$ for all $B \in L^{Y}$.

Proof $(1) \Leftrightarrow(2)$.

$$
\begin{aligned}
& \mathbf{T}_{X}^{*}\left(f^{-1}(B)\right)=\mathbf{T}_{X}\left(f^{-1}\left(B^{*}\right)\right) \geq \mathbf{T}_{Y}\left(B^{*}\right)=\mathbf{T}_{Y}^{*}(B) . \\
& \mathbf{T}_{X}\left(f^{-1}(B)\right)=\mathbf{T}_{X}^{*}\left(f^{-1}\left(B^{*}\right)\right) \geq \mathbf{T}_{Y}^{*}\left(B^{*}\right)=\mathbf{T}_{Y}(B) .
\end{aligned}
$$

(1) $\Rightarrow(3)$. Since $\mathbf{T}_{X}\left(f^{-1}(B)\right) \geq \mathbf{T}_{Y}(B)$ for all $B \in L^{Y}$, we have

$$
\begin{aligned}
& f^{-1}\left(\mathcal{H}_{T_{Y}}\left(\top_{f(x)}, r\right)\right)(y)=\mathcal{H}_{T_{Y}}\left(\top_{f(x)}, r\right)(f(y)) \\
& =\bigwedge\left\{B(f(y)) \mid \top^{\prime} f(x) \leq B, \mathbf{T}_{Y}(B) \geq r^{*}\right\} \\
& \geq \bigwedge\left\{f^{-1}(B)(y) \mid \top_{x} \leq f^{-1}(B), \mathbf{T}_{X}\left(f^{-1}(B)\right) \geq r^{*}\right\} \\
& =\mathcal{H}_{T_{X}}\left(\top_{x}, r\right) .
\end{aligned}
$$

$(3) \Leftrightarrow(5)$. By Theorem 2.8(3) and (3), there exist fuzzy preorders $R_{T_{X}}^{r} \in$ $L^{X \times X}$ and $R_{T_{Y}}^{r} \in L^{Y \times Y}$ with $R_{T_{X}}^{r}(x, y)=\mathcal{H}_{T_{X}}\left(\top_{x}\right)(y)$ and $R_{T_{Y}}^{r}(u, v)=$ $\mathcal{H}_{T_{Y}}\left(\top_{u}\right)(v)$ such that

$$
\begin{aligned}
& f^{-1}\left(\mathcal{H}_{T_{Y}}\left(\top_{f(x)}, r\right)\right)(y) \geq \mathcal{H}_{T_{X}}\left(\top_{x}, r\right)(y) \\
& \text { iff } \left.\mathcal{H}_{T_{Y}}\left(\top_{f(x)}, r\right)\right)(f(y))=R_{T_{Y}}^{r}(f(x), f(y)) \\
& \geq \mathcal{H}_{T_{X}}\left(\top_{x}, r\right)(y)=R_{T_{X}}^{r}(x, y)
\end{aligned}
$$

$(3) \Rightarrow(7)$. By $(3)$, for $B=\bigvee_{z \in Y}\left(B(z) \odot \top_{z}\right)$, we have

$$
\begin{aligned}
& f^{-1}\left(\mathcal{H}_{T_{Y}}(B, r)\right)(y)=\mathcal{H}_{T_{Y}}(B, r)(f(y)) \\
& =\mathcal{H}_{T_{Y}}\left(\bigvee_{z \in Y}\left(B(z) \odot \top_{z}\right), r\right)(f(y)) \\
& =\bigvee_{z \in Y}\left(B(z) \odot \mathcal{H}_{T_{Y}}\left(\top_{z}, r\right)(f(y))\right. \\
& \geq \bigvee_{x \in X}\left(B(f(x)) \odot \mathcal{H}_{T_{Y}}\left(\top_{f(x)}, r\right)(f(y))\right. \\
& \geq \bigvee_{x \in X}\left(\left(f^{-1}(B)(x) \odot \mathcal{H}_{T_{X}}\left(\top_{x}, r\right)(y)\right.\right. \\
& =\mathcal{H}_{T_{X}}\left(f^{-1}(B), r\right)(y) .
\end{aligned}
$$


$(7) \Leftrightarrow(9)$. By $(7)$, put $B=f(A)$. Then

$$
\begin{aligned}
& f^{-1}\left(\mathcal{H}_{T_{Y}}(f(A), r)\right) \geq \mathcal{H}_{T_{X}}\left(f^{-1}(f(A)), r\right) \geq \mathcal{H}_{T_{X}}(A, r) \\
& \text { iff } \mathcal{H}_{T_{Y}}(f(A), r) \geq f\left(\mathcal{H}_{T_{X}}(A, r)\right) .
\end{aligned}
$$

By (9), put $A=f^{-1}(B)$. Then

$$
\begin{aligned}
& \quad f\left(\mathcal{H}_{T_{X}}\left(f^{-1}(B), r\right)\right) \leq \mathcal{H}_{T_{Y}}\left(f\left(f^{-1}(B)\right), r\right) \leq \mathcal{H}_{T_{Y}}(B, r) \\
& \text { iff } \mathcal{H}_{T_{X}}\left(f^{-1}(B), r\right) \leq f^{-1}\left(\mathcal{H}_{T_{Y}}(B, r)\right) . \\
& (7) \Rightarrow(11) .
\end{aligned}
$$

$$
\begin{aligned}
& \mathbf{T}_{X}^{r}\left(f^{-1}(B)\right)=e_{L^{X}}\left(\mathcal{H}_{T_{X}}\left(f^{-1}(B), r\right), f^{-1}(B)\right) \\
& =\bigwedge_{x \in X}\left(\mathcal{H}_{T_{X}}\left(f^{-1}(B), r\right)(x) \rightarrow f^{-1}(B)(x)\right) \\
& \geq \bigwedge_{x \in X}\left(f^{-1}\left(\mathcal{H}_{T_{Y}}(B, r)\right)(x) \rightarrow B(f(x))\right) \\
& \geq \bigwedge_{y \in Y}\left(\mathcal{H}_{T_{Y}}(B, r)(y) \rightarrow B(y)\right) \\
& =\mathbf{T}_{T_{Y}}^{r}(B) .
\end{aligned}
$$

$(11) \Rightarrow(13)$. By $(11)$, since $\mathcal{H}_{T_{Y}}(B, r)=B$ implies $\mathcal{H}_{T_{X}}\left(f^{-1}(B), r\right)=$ $f^{-1}(B)$, the result hold.

$(13) \Leftrightarrow(15)$. Since $\mathbf{T}_{T_{X}}^{r}(A)=e_{L^{X}}\left(\mathcal{H}_{T_{X}}(A, r), A\right)=\top$ iff $A=\mathcal{H}_{T_{X}}(A, r)$, the result holds.

$(15) \Leftrightarrow(1)$. By Theorem $2.8(9)$, it easily proved from $\mathbf{T}_{T_{X}}=\mathbf{T}_{X}$ and $\mathbf{T}_{T_{Y}}=\mathbf{T}_{Y}$.

Other cases are similarly proved.

Example 3.2. Let $\left(L=[0,1], \odot, \rightarrow{ }^{*}\right)$ be a complete residuated lattice with a strong negation defined by

$$
x \odot y=(x+y-1) \vee 0, x \rightarrow y=(1-x+y) \wedge 1, x^{*}=1-x .
$$

Let $X=\{a, b, c, d\}$ and $Y=\{x, y, z\}$ be a set. Define a map $f: X \rightarrow Y$ as

$$
f(a)=f(b)=x, f(c)=y, f(d)=z .
$$

Define fuzzy preorders $R_{X} \in L^{X \times X}$ and $R_{Y} \in L^{Y \times Y}$ as follows:

$$
R_{X}=\left(\begin{array}{cccc}
1 & 0 & 0 & 0 \\
0 & 1 & 0 & 0 \\
0 & 0 & 1 & 0.6 \\
0 & 0 & 0 & 1
\end{array}\right) R_{Y}=\left(\begin{array}{ccc}
1 & 0 & 0 \\
0 & 1 & 0.8 \\
0 & 0 & 1
\end{array}\right)
$$


Then $R_{X}(a, b) \leq R_{Y}(f(a), f(b))$ for all $a, b \in X$. Define

$$
\mathbf{T}_{Y}(B)=\bigwedge_{y \in Y}\left(\bigvee_{x \in X}(B(x) \odot R(x, y)) \rightarrow B(y)\right)
$$

Then $\mathbf{T}_{Y}$ is an Alexandrov fuzzy topology from:

(T1) $\mathbf{T}_{Y}(\bar{\alpha})=\top$,

(T2) By Lemma 2.6 (8), we have

$$
\begin{aligned}
& \mathbf{T}_{Y}\left(\bigwedge_{i \in \Gamma} B_{i}\right)=\bigwedge_{y \in Y}\left(\bigvee_{x \in X}\left(\bigwedge_{i \in \Gamma} B_{i}(x) \odot R(x, y)\right) \rightarrow \bigwedge_{i \in \Gamma} B_{i}(y)\right) \\
& \geq \bigwedge_{i \in \Gamma} \bigwedge_{y \in Y}\left(\bigvee_{x \in X}\left(B_{i}(x) \odot R(x, y)\right) \rightarrow B_{i}(y)\right)=\bigwedge_{i \in \Gamma} \mathbf{T}_{Y}\left(B_{i}\right), \\
& \mathbf{T}_{Y}\left(\bigvee_{i \in \Gamma} B_{i}\right)=\bigwedge_{y \in Y}\left(\bigvee_{x \in X}\left(\bigvee_{i \in \Gamma} B_{i}(x) \odot R(x, y)\right) \rightarrow \bigwedge_{i \in \Gamma} B_{i}(y)\right) \\
& \geq \bigwedge_{i \in \Gamma} \bigwedge_{y \in Y}\left(\bigvee_{x \in X}\left(B_{i}(x) \odot R(x, y)\right) \rightarrow B_{i}(y)\right)=\bigwedge_{i \in \Gamma} \mathbf{T}_{Y}\left(B_{i}\right) .
\end{aligned}
$$

(T3) $\mathbf{T}_{Y}(\alpha \odot B)=\bigwedge_{y \in Y}\left(\bigvee_{x \in X}(\alpha \odot B(x) \odot R(x, y)) \rightarrow \alpha \odot B(y)\right) \geq$ $\bigwedge_{y \in Y}\left(\bigvee_{x \in X}(B(x) \odot R(x, y)) \rightarrow B(y)\right)=\mathbf{T}_{Y}(A)$ from Theorem 2.6 (14).

(T4) $\mathbf{T}_{Y}(\alpha \rightarrow B)=\bigwedge_{y \in Y}\left(\bigvee_{x \in X}((\alpha \rightarrow B)(x) \odot R(x, y)) \rightarrow(\alpha \rightarrow B)(y)\right) \geq$ $\bigwedge_{y \in Y}\left(\bigvee_{x \in X}((\alpha \rightarrow(B(x) \odot R(x, y))) \rightarrow(\alpha \rightarrow B)(y)) \geq \bigwedge_{y \in Y}\left(\bigvee_{x \in X}(B(x) \odot\right.\right.$ $R(x, y)) \rightarrow B(y))=\mathbf{T}_{Y}(A)$ from Theorem $2.6(10,15)$.

$$
\begin{aligned}
& \mathbf{T}_{Y}(B)=\bigwedge_{y \in Y}\left(\bigvee_{x \in X}(B(x) \odot R(x, y)) \rightarrow B(y)\right) \\
& =(B(x) \rightarrow B(x)) \wedge(B(y) \rightarrow B(y)) \wedge((0.8 \odot B(y)) \vee B(z) \rightarrow B(z)) \\
& =(1.2-B(y)+B(z)) \wedge 1 \\
& \mathbf{T}_{X}\left(f^{-1}(B)\right)=\bigwedge_{b \in X}\left(\bigvee_{a \in X}\left(f^{-1}(B)(a) \odot R_{X}(a, b)\right) \rightarrow f^{-1}(B)(b)\right) \\
& =(B(x) \rightarrow B(x)) \wedge(B(y) \rightarrow B(y)) \wedge((0.6 \odot B(y)) \vee B(z) \rightarrow B(z)) \\
& =(1.4-B(y)+B(z)) \wedge 1
\end{aligned}
$$

$$
\begin{aligned}
& \mathbf{T}_{Y}^{*}(B)=\mathbf{T}_{Y}\left(B^{*}\right)=\bigwedge_{y \in Y}\left(\bigvee_{x \in X}\left(B^{*}(x) \odot R(x, y)\right) \rightarrow B^{*}(y)\right) \\
& =\left(B^{*}(x) \rightarrow B^{*}(x)\right) \wedge\left(B^{*}(y) \rightarrow B^{*}(y)\right) \wedge\left(\left(0.8 \odot B^{*}(y)\right) \vee B^{*}(z) \rightarrow B^{*}(z)\right) \\
& =\left(1.2-B^{*}(y)+B^{*}(z)\right) \wedge 1=(1.2-B(z)+B(y)) \wedge 1 . \\
& \mathbf{T}_{Y}^{*}(B)=\bigwedge_{y \in Y}\left(\bigvee_{x \in X}\left(B^{*}(x) \odot R(x, y)\right) \rightarrow B^{*}(y)\right) \\
& =\bigwedge_{x, y \in Y}\left(R(x, y) \rightarrow\left(B^{*}(x) \rightarrow B^{*}(y)\right)\right) \\
& =\bigwedge_{x, y \in Y}(R(x, y) \rightarrow(B(y) \rightarrow B(x))) \\
& =\bigwedge_{x \in Y}\left(\bigvee_{y \in X}(B(y) \odot R(x, y)) \rightarrow B(x)\right) \\
& \mathbf{T}_{X}^{*}\left(f^{-1}(B)\right)=\bigwedge_{b \in X}\left(\bigvee_{a \in X}\left(f^{-1 *}(B)(a) \odot R_{X}(a, b)\right) \rightarrow f^{-1 *}(B)(b)\right) \\
& =\left(B^{*}(x) \rightarrow B^{*}(x)\right) \wedge\left(B^{*}(y) \rightarrow B^{*}(y)\right) \wedge\left(\left(0.6 \odot B^{*}(y)\right) \vee B^{*}(z) \rightarrow B^{*}(z)\right) \\
& =\left(1.4-B^{*}(y)+B^{*}(z)\right) \wedge 1=(1.4-B(z)+B(y)) \wedge 1 .
\end{aligned}
$$

Hence $\mathbf{T}_{X}\left(f^{-1}(B)\right) \geq \mathbf{T}_{Y}(B)$ and $\mathbf{T}_{X}^{*}\left(f^{-1}(B)\right) \geq \mathbf{T}_{Y}^{*}(B)$ for all $B \in L^{Y}$ and $r \in L$. 
Since $\mathcal{H}_{T_{Y}}\left(1_{y}, r\right)(z)=\bigwedge\left\{B(z) \in L^{X} \mid 1_{y} \leq B, \mathbf{T}_{Y}(B) \geq r^{*}\right\}$, then $\mathcal{H}_{T_{Y}^{*}}\left(1_{y}, r\right)(z)=\mathcal{H}_{T_{Y}}\left(1_{z}, r\right)(y)$ from:

$$
\begin{array}{ccc}
\mathcal{H}_{T_{Y}}\left(1_{x}, r\right)(x)=1 & \mathcal{H}_{T_{Y}}\left(1_{x}, r\right)(y)=0 & \mathcal{H}_{T_{Y}}\left(1_{x}, r\right)(z)=0 \\
\mathcal{H}_{T_{Y}}\left(1_{y}, r\right)(x)=0 & \mathcal{H}_{T_{Y}}\left(1_{y}, r\right)(y)=1 & \mathcal{H}_{T_{Y}}\left(1_{y}, r\right)(z)=(0.8-r) \vee 0 \\
\mathcal{H}_{T_{Y}}\left(1_{z}, r\right)(x)=0 & \mathcal{H}_{T_{Y}}\left(1_{z}, r\right)(y)=0 & \mathcal{H}_{T_{Y}}\left(1_{z}, r\right)(z)=1 \\
& & \\
\mathcal{H}_{T_{Y}^{*}}\left(1_{x}, r\right)(x)=1 & \mathcal{H}_{T_{Y}^{*}}\left(1_{x}, r\right)(y)=0 & \mathcal{H}_{T_{Y}^{*}}\left(1_{x}, r\right)(z)=0 \\
\mathcal{H}_{T_{Y}^{*}}\left(1_{y}, r\right)(x)=0 & \mathcal{H}_{T_{Y}^{*}}\left(1_{y}, r\right)(y)=1 & \mathcal{H}_{T_{Y}^{*}}\left(1_{y}, r\right)(z)=0 \\
\mathcal{H}_{T_{Y}^{*}}\left(1_{z}, r\right)(x)=0 & \mathcal{H}_{T_{Y}^{*}}\left(1_{z}, r\right)(y)=(0.8-r) \vee 0 & \mathcal{H}_{T_{Y}^{*}}\left(1_{z}, r\right)(z)=1 .
\end{array}
$$

Similarly, we have $\mathcal{H}_{T_{X}^{*}}\left(1_{a}, r\right)(b)=\mathcal{H}_{T_{X}}\left(1_{b}, r\right)(a)$ for all $a, b \in X$ as

$$
\left(\mathcal{H}_{T_{X}}\left(1_{a}, r\right)(b)\right)=\left(\begin{array}{cccc}
1 & 0 & 0 & 0 \\
0 & 1 & 0 & 0 \\
0 & 0 & 1 & (0.6-r) \vee 0 \\
0 & 0 & 0 & 1
\end{array}\right)
$$

Hence $f^{-1}\left(\mathcal{H}_{T_{Y}}\left(\top_{f(a)}, r\right)\right) \geq \mathcal{H}_{T_{X}}\left(\top_{a}, r\right)$ and $f^{-1}\left(\mathcal{H}_{T_{Y}^{*}}\left(\top_{f(a)}, r\right)\right) \geq \mathcal{H}_{T_{X}^{*}}\left(\top_{a}, r\right)$ for all $x \in X$ and $r \in L$. Since $R_{T_{Y}}^{r}(x, y)=\mathcal{H}_{T_{Y}}\left(\top_{x}, r\right)(y) R_{T_{X}}^{r}(a, b)=$ $\mathcal{H}_{T_{X}}\left(\top_{a}, r\right)(b)$, we have

$$
R_{T_{Y}^{*}}^{r}(x, y)=R_{T_{Y}}^{r}(y, x), R_{T_{X}^{*}}^{r}(a, b)=R_{T_{X}}^{r}(b, a) .
$$

Moreover, for all $a, b \in X$,

$$
\begin{gathered}
R_{T_{X}}^{r}(a, b) \leq R_{T_{Y}}^{r}(f(a), f(b)), \quad R_{T_{X}^{*}}^{r}(a, b) \leq R_{T_{Y}^{*}}^{r}(f(a), f(b)) . \\
R_{T_{X}}^{r}=\left(\begin{array}{cccc}
1 & 0 & 0 & 0 \\
0 & 1 & 0 & 0 \\
0 & 0 & 1 & (0.6-r) \vee 0 \\
0 & 0 & 0 & 1
\end{array}\right) \quad R_{T_{Y}}^{r}=\left(\begin{array}{ccc}
1 & 0 & 0 \\
0 & 1 & (0.8-r) \vee 0 \\
0 & 0 & 1
\end{array}\right)
\end{gathered}
$$

For $B=\bigvee_{x \in Y}\left(B(x) \odot 1_{x}\right)$, we have

$$
\begin{aligned}
& \mathcal{H}_{T_{Y}}(B, r)=\mathcal{H}_{T_{Y}}\left(\bigvee_{x \in Y}\left(B(x) \odot 1_{x}\right), r\right) \\
& =\bigvee_{x \in Y}\left(B(x) \odot \mathcal{H}_{T_{Y}}\left(1_{x}, r\right)\right)=\bigvee_{x \in Y}\left(B(x) \odot R_{T_{Y}}^{r}(x,-)\right) \\
& =(B(x), B(y),(B(y)-0.2-r) \vee B(z)) . \\
& \mathcal{H}_{T_{Y}^{*}}(B, r)(y)=\mathcal{H}_{T_{Y}^{*}}\left(\bigvee_{x \in Y}\left(B(x) \odot 1_{x}\right), r\right)(y) \\
& =\bigvee_{x \in Y}\left(B(x) \odot \mathcal{H}_{T_{Y}^{*}}\left(1_{x}, r\right)\right)=\bigvee_{x \in Y}\left(B(x) \odot R_{T_{Y}}^{r}(-, x)\right) \\
& =(B(x), B(y) \vee(B(z)-0.2-r), B(z)) .
\end{aligned}
$$




$$
\begin{aligned}
& \mathcal{H}_{T_{X}}(A, r)=(A(a), A(b), A(c),(A(c)-0.4-r) \vee A(d)), \\
& \mathcal{H}_{T_{X}^{*}}(A, r)=(A(a), A(b), A(c) \vee(A(d)-0.4-r), A(d)) . \\
& \left.\quad \mathcal{H}_{T_{X}}\left(f^{-1}(B), r\right)\right)=\bigvee_{a \in X}\left(f^{-1}(B)(a) \odot R_{T_{X}}^{r}(a,-)\right) \\
& \quad=(B(x), B(x), B(y),(B(y)-0.4-r) \vee B(z)) \\
& \quad \leq f^{-1}\left(\mathcal{H}_{T_{X}}(B, r)\right)=f^{-1}\left(\bigvee_{x \in Y}\left(B(x) \odot R_{T_{Y}}^{r}(x,-)\right)\right. \\
& \quad=(B(x), B(x), B(y),(B(y)-0.2-r) \vee B(z)) . \\
& \left.\quad \mathcal{H}_{T_{X}^{*}}\left(f^{-1}(B), r\right)\right)=\bigvee_{a \in X}\left(f^{-1}(B)(a) \odot R_{T_{X}}^{r}(-, a)\right) \\
& \quad=(B(x), B(x), B(y) \vee(B(z)-0.4-r), B(z)) \\
& \quad \leq f^{-1}\left(\mathcal{H}_{T_{X}^{*}}(B, r)\right)=f^{-1}\left(\bigvee_{x \in Y}\left(B(x) \odot R_{T_{Y}}^{r}(-, x)\right)\right. \\
& \quad=(B(x), B(x), B(y) \vee(B(z)-0.2-r), B(z)) .
\end{aligned}
$$

$$
\begin{aligned}
& \mathbf{T}_{T_{Y}}^{r}(B)=\bigwedge_{y \in Y}\left(\mathcal{H}_{T_{Y}}(B, r)(y) \rightarrow B(y)\right) \\
& =((B(y)-0.2-r) \vee B(z)) \rightarrow B(z)=(1.2+r-B(y)+B(z)) \wedge 1 \\
& \mathbf{T}_{T_{X}}^{r}(A)=\bigwedge_{a \in X}\left(\mathcal{H}_{T_{X}}(A, r)(a) \rightarrow A(a)\right) \\
& =((A(c)-0.4-r) \vee A(d)) \rightarrow A(d)=(1.4+r-A(c)+A(d)) \wedge 1 \\
& \mathbf{T}_{T_{Y}^{*}}^{r}(B)=\bigwedge_{y \in Y}\left(\mathcal{H}_{T_{Y}^{*}}(B, r)(y) \rightarrow B(y)\right) \\
& =(B(y) \vee(B(z)-0.2-r)) \rightarrow B(y)=(1.2+r-B(z)+B(y)) \wedge 1 \\
& \begin{aligned}
\mathbf{T}_{T_{X}^{*}}^{r}(A)= & \bigwedge_{a \in X}\left(\mathcal{H}_{T_{X}^{*}}(A, r)(a) \rightarrow A(a)\right) \\
=(A(c) \vee & (A(d)-0.4-r)) \rightarrow A(c)=(1.4+r-A(d)+A(c)) \wedge 1 \\
& \mathbf{T}_{T_{X}}^{r}\left(f^{-1}(B)\right)=(1.4+r-B(y)+B(z)) \wedge 1 \\
& \mathbf{T}_{T_{X}^{*}}^{r}\left(f^{-1}(B)\right)=(1.4+r-B(z)+B(y)) \wedge 1
\end{aligned}
\end{aligned}
$$

Hence $\mathbf{T}_{T_{X}}^{r}\left(f^{-1}(B)\right) \geq \mathbf{T}_{T_{Y}}^{r}(B)$ and $\mathbf{T}_{T_{X}^{*}}^{r}\left(f^{-1}(B)\right) \geq \mathbf{T}_{T_{X}^{*}}^{r}(B)$ for all $B \in L^{Y}$ and $r \in L$.

$$
\begin{aligned}
& \mathbf{T}_{H_{T_{Y}}}(B)=\bigvee\left\{r^{*} \in L \mid \mathcal{H}_{T_{Y}}(B, r)=B\right\} \\
& =\bigvee\left\{r^{*} \in L \mid(B(x), B(y),(B(y)-0.2-r) \vee B(z))=(B(x), B(y), B(z))\right\} \\
& =\bigvee\left\{r^{*} \in L \mid B(y)-0.2-r \leq B(z)\right\}=(1.2-B(y)+B(z)) \wedge 1=\mathbf{T}_{Y}(B) \\
& \quad \mathbf{T}_{H_{T_{X}}}\left(f^{-1}(B)\right)=\bigvee\left\{r^{*} \in L \mid \mathcal{H}_{T_{Y}}\left(f^{-1}(B), r\right)=f^{-1}(B)\right\} \\
& =\bigvee\left\{r^{*} \in L \mid B(y)-0.4-r \leq B(z)\right\}=(1.4-B(y)+B(z)) \wedge 1 .
\end{aligned}
$$

Hence $\mathbf{T}_{H_{T_{X}}}\left(f^{-1}(B)\right) \geq \mathbf{T}_{H_{T_{Y}}}(B)$ for all $B \in L^{Y}$ and $r \in L$. 


$$
\begin{aligned}
\mathbf{T}_{T_{Y}}(B) & =\bigvee\left\{r^{*} \in L \mid \mathbf{T}_{T_{Y}}^{r}(B)=(1.2+r-B(y)+B(z)) \wedge 1=1\right\} \\
& =\left(1.2-B(y)+B(z) \wedge 1=\mathbf{T}_{Y}(B),\right. \\
\mathbf{T}_{T_{Y}^{*}}(B) & =\bigvee\left\{r^{*} \in L \mid \mathbf{T}_{T_{Y}^{*}}^{r}(B)=(1.2+r-B(z)+B(y)) \wedge 1=1\right\} \\
& =(1.2-B(z)+B(y)) \wedge 1=\mathbf{T}_{Y}^{*}(B) . \\
\mathbf{T}_{T_{X}}\left(f^{-1}(B)\right) & =\bigvee\left\{r^{*} \in L \mid \mathbf{T}_{T_{X}}^{r}\left(f^{-1}(B)\right)=(1.4+r-B(y)+B(z)) \wedge 1=1\right\} \\
=(1.4-B(y) & +B(z) \wedge 1=\mathbf{T}_{X}\left(f^{-1}(B)\right), \\
\mathbf{T}_{T_{X}^{*}}\left(f^{-1}(B)\right) & =\bigvee\left\{r^{*} \in L \mid \mathbf{T}_{T_{X}^{*}}^{r}\left(f^{-1}(B)\right)=(1.4+r-B(z)+B(y)) \wedge 1=1\right\} \\
=(1.4-B(z) & +B(y)) \wedge 1=\mathbf{T}_{X}^{*}\left(f^{-1}(B)\right) .
\end{aligned}
$$

Hence $\mathbf{T}_{T_{X}}\left(f^{-1}(B)\right) \geq \mathbf{T}_{T_{Y}}(B)$ and $\mathbf{T}_{T_{X}^{*}}\left(f^{-1}(B)\right) \geq \mathbf{T}_{T_{Y}^{*}}(B)$ for all $B \in L^{Y}$ and $r \in L$.

\section{References}

[1] R. Bělohlávek,Fuzzy Relational Systems, Kluwer Academic Publishers, New York , (2002), doi: 10.1007/978-1-4615-0633-1.

[2] P. Hájek, Metamathematices of Fuzzy Logic, Kluwer Academic Publishers, Dordrecht (1998), doi: 10.1007/978-94-011-5300-3.

[3] U. Höhle, S.E. Rodabaugh, Mathematics of Fuzzy Sets: Logic, Topology, and Measure Theory, The Handbooks of Fuzzy Sets Series 3, Kluwer Academic Publishers, Boston (1999).

[4] Fang Jinming, I-fuzzy Alexandrov topologies and specialization orders, Fuzzy Sets and Systems, 158(2007), 2359-2374, doi: 10.1016/j.fss.2007.05.001.

[5] Y.C. Kim, Join preserving maps, fuzzy preorders and Alexandrov fuzzy topologies, International Journal of Pure and Applied Mathematics, 92, No.5(2014), 703-718, doi: 10.12732/ijpam.v92i5.6.

[6] Y.C. Kim, Upper approximation oprators induced by Alexandrov fuzzy topologies, International Journal of Pure and Applied Mathematics, 92,No.5(2014), 719-729, doi: 10.12732/ijpam.v92i5.7.

[7] Y.C. Kim, Upper approximation oprators induced by maps, International Journal of Pure and Applied Mathematics, 92, No. 5(2014), 731-744, doi: 10.12732/ijpam.v92i5.8. 
[8] J. Kortelainen, On relationships between modified sets, topological spaces and rough sets, Fuzzy Sets and Systems, 61(1994), 91-95, doi: 10.1016/0165-0114(94)90288-7.

[9] H. Lai, D. Zhang, Fuzzy preorder and fuzzy topology, Fuzzy Sets and Systems, 157 (2006), 1865-1885, doi: 10.1016/j.fss.2006.02.013.

[10] H. Lai, D. Zhang, Concept lattices of fuzzy contexts: Formal concept analysis vs. rough set theory, Int. J. Approx. Reasoning, 50 (2009), 695-707, doi: $10.1016 /$ j.ijar.2008.12.002.

[11] Z. Pawlak, Rough sets, Int. J. Comput. Inf. Sci., 11 (1982), 341-356, , doi: 10.1007/BF01001956.

[12] Z. Pawlak, Rough probability, Bull. Pol. Acad. Sci. Math., 32(1984), 607615.

[13] A. M. Radzikowska, E.E. Kerre, A comparative study of fuzy rough sets, Fuzzy Sets and Systems, 126(2002), 137-155, doi: 10.1016/so1650114(01)00032-x.

[14] S. P. Tiwari, A.K. Srivastava, Fuzzy rough sets, fuzzy preorders and fuzzy topologies, Fuzzy Sets and Systems, 210(2013), 63-68, doi: 10.1016/j.fss.2012.06.001.

[15] Y.H. She, G.J. Wang, An axiomatic approach of fuzzy rough sets based on residuated lattices, Computers and Mathematics with Applications, $\mathbf{5 8}$ (2009), 189-201, doi: 10.1016/j.camwa.2009.03.100.

[16] Zhen Ming Ma, Bao Qing Hu, Topological and lattice structures of Lfuzzy rough set determined by lower and upper sets, Information Sciences, 218(2013), 194-204, doi: 10.1016/j.ins.2012.06.029. 
